\title{
Skin friction in zero-pressure-gradient boundary layers.
}

\author{
Victor Yakhot* \\ Department of Mechanical Engineering, \\ Boston University, Boston, MA 02215
}

(Dated: today)

\begin{abstract}
A global approach leading to a self-consistent solution to the Navier-Stokes-Prandtl equations for zero-pressure-gradient boundary layers is presented. It is shown that as $R e_{\delta} \rightarrow \infty$, the dynamically defined boundary layer thickness $\delta(x) \propto x / \ln ^{2} R e_{x}$ and the skin friction $\lambda=\frac{2 \tau_{w}}{\rho U_{0}^{2}} \propto 1 / \ln ^{2} \delta(x)$. Here $\tau_{w}$ and $U_{0}$ are the wall shear stress and free stream velocity, respectively. The theory is formulated as an expansion in powers of a small dimensionless parameter $\frac{d \delta(x)}{d x} \rightarrow 0$ in the limit $x \rightarrow \infty$.
\end{abstract}

The law of variation of skin friction with Reynolds number in turbulent wall flows is one of the oldest riddles of physics of turbulence. In addition to difficulties associated with a general problem of strong isotropic turbulence, the presence of solid walls is responsible for appearance of two different characteristic velocities. The so called friction velocity, reflecting properties of the nearwall sublayer, is equal to $u_{*}^{2}=\nu\left|\frac{\partial U(y)}{\partial y}\right|_{\text {wall }}$ and the externally prescribed free-stream velocity is denoted as $U_{0}$. For dimensionless distance to the wall $y_{+}=\frac{y u_{*}}{\nu}=O(1)$, the dimensionless velocity profile $U_{+}\left(y_{+}\right)=U\left(y_{+}\right) / u_{*} \propto$ $y_{+}$is independent upon the Reynolds number. It is the interplay of the two characteristic velocities that makes derivation of mean velocity distributions in wall flows so difficult.

The first semi-empirical theory of zero-pressure-gradient boundary layer (BL) was developed by Prandtl (for detailed description see Ref.[1]) and references therein) who, defining self-similar variables $U(x, y)=U\left(\frac{y}{\delta(x)}\right)$, introduced the boundary layer thickness $\delta(x)$ depending upon distance to the origin $x$. In addition, from the force balance, Prandtl obtained the expression for skin friction $\lambda \propto \frac{d \delta}{d x} \propto\left(\frac{u_{*}}{U_{0}}\right)^{2}$. Then, as a stroke of genius, based on experimental data on skin friction in pipes and channels $\lambda_{\text {pipe }} \propto R e_{D}^{-\frac{1}{4}}$ available in those early days, he, identifying $\delta(x)$ with the pipe diameter $D$, proposed the differential relation:

$$
\frac{d \delta(x)}{d x} \propto\left(\frac{\nu}{\delta U_{0}}\right)^{\frac{1}{4}}
$$

giving $\delta(x) \propto \frac{x}{R e_{x}^{0.2}}$, and $\lambda \propto R e_{x}^{-0.2}$ - the expressions often used in modern engineering literature and textbooks. A different, more detailed, formulation was based on a a force balance following the equations of motion:

$$
\lambda \propto \frac{d \delta(x)}{d x} \int_{0}^{\delta(x)} \frac{U(y)}{U_{0}}\left(1-\frac{U(y)}{U_{0}}\right) d y
$$

\footnotetext{
*Electronic address: vy@bu.edu
}

Then substituting into this expression the assumed velocity profile $\frac{U(y)}{U_{0}} \propto \phi\left(\frac{y}{\delta(x)}\right)$ with either algebraic or logarithmic expressions for the function $\phi(z)$ led to different relations for skin frictions reasonably well representing experimental data in a restricted range of Reynolds number variation. Due to the lack of mathematically sound derivation, even today, the question of "correct" velocity distribution in fully developed pipes/channel flows is a topic of lively scientific discussions. These early theories, based on bold assumptions combined with deep physical insights, led to helpful expressions widely used in engineering.

The modern analysis of pipe/channel and boundary layers is typically based on an assumed scaling relation for velocity represented in the "inner" and "outer" regions of the flow as $U(y)=u_{*} f\left(y_{+}\right)$and $U(y)=U_{c L}-u_{0} g\left(\frac{y}{H}\right)$, respectively ${ }^{2}$. The parameters $u_{*}$ and $u_{0}$ are corresponding characteristic velocities. Then, different matching conditions applied to the "overlap" region lead to different shapes of velocity profile $U(y)$. According to experimental data ${ }^{2}$, in the limit $R e_{D} \rightarrow \infty$, the second characteristic velocity $u_{0} \rightarrow u_{*}$. In a recent paper ${ }^{3}$ assuming the logarithmic velocity profile across a zeropressure-gradient boundary layer, developed an asymptotic expansion, leading to the so called Coles-Fernholtz relation ${ }^{4}$ :

$$
\lambda \propto \frac{1}{\ln ^{2} \operatorname{Re}_{\delta(x)}}
$$

widely accepted as an accurate large Reynolds number ( $R e_{D}>>10^{5}$ ) asymptotics. While this work is based on a solid mathematical analysis, its starting point, logarithmic profile, is an assumption not following the NavierStokes equations.

The theory developed below is based on the following observation. It is well known that in the small-parameterlacking homogeneous and isotropic turbulence evaluation of moments of velocity fluctuations is an extremely difficult and unsolved problem. In this regard the problem of statistical properties of wall turbulence is at least as difficult. However, in wall flows as $R e_{\delta} \rightarrow \infty$, the 
global dimensionless parameters $u_{*} / \bar{U} \rightarrow 0$ and $\frac{d \delta(x)}{d x} \rightarrow 0$ are small and can be used for construction of the wellbehaved perturbation expansion leading to prediction of global properties of wall flows. This is the main goal of this paper.

We consider a flat plate $0 \leq x \leq \infty$ and $y=0$. The freestream velocity of incoming flow is $\mathbf{U}_{0}=U_{0} \mathbf{i}$ and we are to analyze the Navier-Stokes -Prandtl equations in the boundary layer approximation:

$$
\begin{gathered}
\frac{\partial U}{\partial x}+\frac{\partial V}{\partial y}=0 \\
U \frac{\partial U}{\partial x}+V \frac{\partial U}{\partial y}=\frac{\partial}{\partial y}\left(\nu \frac{\partial U}{\partial y}+\tau_{x, y}\right)
\end{gathered}
$$

with $\tau_{x, y}=-\overline{u_{x} u_{y}}$ and the mean is taken over ensemble of fluctuating velocity field $\mathbf{u}$. Interested in the global, integral, features of a flow we neglect contribution coming from extremely thin in the limit $\operatorname{Re}_{\delta} \rightarrow \infty$ viscous sublayer $0 \leq y \leq y_{s L} \rightarrow 0$ and as $x \rightarrow \infty$ we, assuming self-similarity of velocity profile write: $U(x, y)=$ $U\left(x, \frac{y}{\delta(x)}\right) \equiv U(x, \eta), V=V\left(x, \frac{y}{\delta(x)}\right) \equiv V(x, \eta)$ and $\tau_{x, y}=\tau_{x, y}\left(x, \frac{y}{\delta(x)}\right) \equiv \tau_{x, y}(x, \eta)$ where the defined below width of the boundary layer $\delta(x)$ must be found from equations of motion. The incompressibility constraint (2) gives:

$$
\begin{array}{r}
V(x, y)=-\int_{0}^{y} \frac{\partial U\left(x, y^{\prime}\right)}{\partial x} d y^{\prime}= \\
-\delta \int_{0}^{\eta} \frac{\partial U\left(x, \eta^{\prime}\right)}{\partial x} d \eta^{\prime}+\frac{\partial \delta(x)}{\partial x} \int_{0}^{\eta} \eta^{\prime} \frac{d U\left(x, \eta^{\prime}\right)}{d \eta^{\prime}} d \eta^{\prime}
\end{array}
$$

Integrating (3) over the interval $0 \leq y \leq \infty$, and introducing the 'displacement thickness' $\theta$ we, using (4), express the skin friction in terms of the boundary layer thickness $\delta$ :

$$
\begin{gathered}
\frac{d \theta}{d x}=\int_{0}^{\infty} G(x, \eta)\left(\frac{\delta}{U_{0}} \frac{\partial U(x, \eta)}{\partial x}+\frac{\partial \delta(x)}{\partial x} \frac{U(x, \eta)}{U_{0}}\right) d \eta= \\
=\frac{u_{*}^{2}}{U_{0}^{2}}=\lambda / 2
\end{gathered}
$$

where $u_{*}^{2}(x)=\left.\nu \frac{\partial U(x, y)}{\partial y}\right|_{0}$ and $G(x, \eta)=1-\frac{U(x, \eta)}{U_{0}}$. In this work we are interested in the global, integral, features of wall turbulence, like $\bar{U}$ with the negligible contribution from the viscous sublayer $y / \delta<y_{s L} / \delta \rightarrow 0$ in the limit $\operatorname{Re}_{\delta} \rightarrow \infty$. Therefore, in what follows we seek solution in the form

$$
U(x, y)=u_{*}(x) \varphi(\eta)
$$

so that the relation (5) becomes:

$$
\begin{array}{r}
\delta\left(\frac{d}{d x} \frac{u_{*}}{U_{0}}\right) \int_{0}^{\infty} G(x, \eta) \varphi(\eta) d \eta+ \\
\frac{\partial \delta(x)}{\partial x} \frac{u_{*}(x)}{U_{0}} \int_{0}^{\infty} \varphi(\eta) G(x, \eta) d \eta \\
=\frac{u_{*}^{2}}{U_{0}^{2}} \equiv i_{1}+i_{2}=\lambda / 2
\end{array}
$$

It will become clear below that in the limit $R e_{x}=$ $x U_{0} / \nu \rightarrow \infty$ the first term in the right side of (5) is negligibly small and therefore $\lambda=2\left(\frac{u_{*}}{U_{0}}\right)^{2} \propto \frac{d \delta}{d x} \rightarrow 0$. Based on as yet unknown function $\delta(x)$, we define an averaged- over- the -boundary -layer property $\Psi: \overline{\Psi(x)}=$ $\frac{1}{\delta} \int_{0}^{\delta} \Psi(x, y) d y=\int_{0}^{1} \Psi(x, \eta) d \eta$. Since at the edge of a boundary layer $y=\delta(x)$, the velocity and kinetic energy are $U=U(x, \delta(x))$ and $K=K(x, \delta(x))$, respectively, the familiar integral balance equations must be somewhat modified. For example, integrating the differential energy balance equation:

$$
U \frac{\partial K}{\partial x}+V \frac{\partial K}{\partial y}=-\tau_{x y} \frac{\partial U}{\partial y}-\mathcal{E}+\frac{\partial}{\partial y}\left(\nu \frac{\partial K}{\partial y}+Q\right)
$$

in the interval $0 \leq y \leq \delta(x)$ and recalling that

$$
\int_{0}^{\delta} V \frac{\partial K}{\partial y} d y=V(\delta) K(\delta)-\int_{0}^{\delta} K \frac{\partial V}{\partial y} d y
$$

we, using an incompressibility constraint, derive:

$$
\begin{array}{r}
\int_{0}^{\delta} \frac{d}{d x} K(x, y) U(x, y) d y+V(x, \delta) K(\delta)= \\
-\int_{0}^{\delta} \tau_{x y} \frac{\partial U}{\partial y} d y-\delta \overline{\mathcal{E}}+Q(\delta)
\end{array}
$$

where $Q(\delta(x))=\overline{w(\delta) u_{i}^{2}(\delta)}=O\left(u_{*}^{3}\right)$. In the limit $\delta \rightarrow$ $\infty, V(x, \delta) \rightarrow 0$ and the relation (8) tends to a familiar energy balance (see for example Ref.[5]). With

$$
\begin{array}{r}
V(x, \delta(x))=-\delta \frac{d \bar{U}(x)}{d x}+\frac{d \delta}{d x}(U(x, \delta)-\bar{U}(x))= \\
-\frac{d}{d x}(\delta \bar{U}(x))+\frac{d \delta}{d x} U(x, \delta)
\end{array}
$$

directly following from (4), the relation (8) takes a very simple form:

$$
\frac{d}{d x}(\delta \overline{K U})-K(x, \delta) \frac{d}{d x}(\delta \bar{U})=\int_{0}^{\delta} \tau_{x y} \frac{\partial U}{\partial y} d y-\delta \overline{\mathcal{E}}+Q(\delta)
$$

The left side of (10) is the energy balance in the crosssection $x$. As $R e_{x} \rightarrow \infty$ we can define $K_{0}(x)$ so that the mean: 


$$
\overline{K U}=\frac{1}{\delta} \int_{0}^{\delta(x)} K(x, y) U(x, y) d y=K_{0}(x) \bar{U}(x)
$$

In the bulk of the flow $y \gg y_{s L} \rightarrow 0$ dominating the integral (11), where the turbulent kinetic energy $K(x, y)$ is a weak function of $y$, the parameter $(\bar{K}(x)-$ $\left.K_{0}(x)\right) / \overline{K(x)} \ll 1$.

Expansion. Now, recalling that $u_{*}=u_{*}(x)$ depends on the distance to the origin $x$, we introduce a definition of the boundary layer thickness:

$$
\begin{array}{r}
U(\delta(x))-\bar{U}(x) \propto U_{0}-\bar{U}(x)=u_{*} \phi_{u}\left(\frac{u_{*}}{U_{0}}\right) \\
K_{0}(x)=u_{*}^{2} \phi_{K}\left(\left(\frac{u_{*}}{U_{0}}\right)^{2}\right) \\
K(\delta(x))=u_{*}^{2} \phi\left(\left(\frac{u_{*}}{U_{0}}\right)^{2}\right)
\end{array}
$$

As $R e_{\delta} \rightarrow \infty$ the dimensionless parameter $\xi(x)=$ $\frac{u_{*}(x)}{U_{0}} \rightarrow 0$ and taking into account that in this limit all functional derivatives

$$
\frac{d^{n} U_{+}}{d \xi^{n}} ; \quad \frac{d^{n} \frac{K_{0}}{u_{*}^{2}}}{d \xi^{n}}
$$

are finite (for a discussion see below), we derive:

$$
\begin{aligned}
U(\delta(x))-\bar{U}(x) \propto U_{0}-\bar{U}(x) & \approx u_{*} \sum_{n=0}^{\infty} \alpha_{n}\left(\frac{u_{*}}{U_{0}}\right)^{n} \\
K_{0}(x) & =u_{*}^{2} \sum_{n=0}^{\infty} b_{n}\left(\frac{u_{*}}{U_{0}}\right)^{2 n} \\
K(\delta(x)) & \approx u_{*}^{2} \sum_{n=0}^{\infty} c_{n}\left(\frac{u_{*}}{U_{0}}\right)^{2 n}
\end{aligned}
$$

where $K_{0}(x)$ is defined as:

$\overline{K U}=K_{0}(x) \bar{U}(x)=u_{*}^{2}\left(U_{0}-u_{*} \sum_{i=0}^{\infty} \alpha_{i}\left(\frac{u_{*}}{U_{0}}\right)^{i}\right) \times \sum_{n=0}^{\infty} b_{n}\left(\frac{u_{*}}{U_{0}}\right)^{2 n}$

The relations (14) is a definition of expansion coefficients $b_{n}$. Let us demonstrate that the anzatz (12)-(13), combined with the energy balance (10), leads the well-known empirical relation $\lambda \propto \frac{1}{\ln ^{2} R e_{\delta}}$. Neglecting for a time being the contribution $i_{1}$ to (7) as small (it will be justified below) we obtain simple estimates:

$$
\frac{d \delta}{d x} u_{*}^{2} U_{0}=O\left(\frac{u_{*}^{4}}{U_{0}}\right) \ll u_{*}^{3} ; \quad \frac{d \delta}{d x} u_{*}^{3}=O\left(\frac{u_{*}^{5}}{U_{0}^{2}}\right) \ll \frac{u_{*}^{4}}{U_{0}} ;
$$

It will become clear below that as $x \rightarrow \infty, \delta u_{*}^{2} \frac{d u_{*}}{d x}=$ $O\left(u_{*}^{5} / U_{0}^{2}\right)$. Substituting the anzatz (12)-(13) into the energy balance (10) and taking into account the estimates (15), we, equating the terms of equal powers of small parameter $u_{*} / U_{0}$ obtain in the zeroth order:

$$
\int_{0}^{\delta} \tau_{x y} \frac{\partial U}{\partial y} d y=\delta \overline{\mathcal{E}}+Q(\delta)=O\left(u_{*}^{3}\right)
$$

Locally, the dissipation rate $\mathcal{E}$ satisfies a well - known universal scaling relation $\mathcal{E}(y)=\frac{u_{*}^{4}}{\nu} \mathcal{E}_{+}\left(\frac{y u_{*}}{\nu}\right)=\frac{u_{*}^{4}}{\nu} \mathcal{E}_{+}\left(y_{+}\right)$ which has recently been tested experimentally in pipe flows ${ }^{6-7}$. Thus:

$$
\delta \overline{\mathcal{E}}=u_{*}^{3} \int_{0}^{\delta} \mathcal{E}_{+}\left(y_{+}\right) d \frac{y u_{*}}{\nu}=u_{*}^{3} \int_{0}^{R_{*}} \mathcal{E}_{+}\left(y_{+}\right) d y_{+}=O\left(u_{*}^{3}\right)
$$

justifying the above zero-order estimate, provided in the limit $R_{*} \rightarrow \infty$ the function $\mathcal{E}_{+}$decreases with $y_{+}$rapidly enough so that the convergent integral is independent of the upper limit. The production term is:

$$
\begin{array}{r}
\int_{0}^{\delta} \tau_{x y} \frac{\partial U}{\partial y} d y= \\
u_{*}^{2} \int_{0}^{1} \tau_{+} \frac{d U(\eta)}{d \eta} d \eta \approx-u_{*}^{3} \int_{0}^{1} \tau_{+} \frac{d g(\eta)}{d \eta} d \eta=O\left(u_{*}^{3}\right)
\end{array}
$$

$\left(\tau_{+}=\tau_{x y} / u_{*}^{2}\right)$ where in the limit $R e_{\delta} \rightarrow \infty$ the integral is dominated by the outer scaling $U(y)=U_{0}-u_{*} g(y / \delta)$ (Smits (1999)). The flux contribution $Q(\delta)=O\left(u_{*}^{3}\right)$.

According to this estimate, contributions to the right and left side of (10) are balanced separately. This fact becomes clear if we notice that each term in the left side of (10) involves $x$-derivatives which are proportional to $\frac{d \delta}{d x} \propto\left(\frac{u_{*}}{U_{0}}\right)^{2} \rightarrow 0$ while each contribution to the right side is $O\left(u_{*}^{3}\right)=O(1)$. Moreover, since in the interval $y>\delta$, all turbulence characteristics sharply decrease to zero, all integrals over the $y$-coordinate in the right side are convergent so that the outcome is independent neither of the upper limit $\delta$ nor $\frac{d \delta}{d x}$. The remaining terms are:

$$
\begin{aligned}
\frac{d \delta}{d x}\left[u_{*}^{2}\left(U_{0}-u_{*} \sum_{n=0}^{\infty} \alpha_{n}\left(\frac{u_{*}}{U_{0}}\right)^{n}\right) \times \sum_{i=0}^{\infty}\left(b_{i}-c_{i}\right)\left(\frac{u_{*}}{U_{0}}\right)^{2 i}\right]+ \\
\left.\delta(x) u_{*}^{2} \sum_{n=0}^{\infty} c_{n}\left(\frac{u_{*}}{U_{0}}\right)^{2 n} \times \frac{d}{d x}\left(u_{*} \sum_{i} \alpha_{i}\left(\frac{u_{*}}{U_{0}}\right)^{i}\right)=\emptyset 16\right)
\end{aligned}
$$

We see that the first -order in $u_{*} / U_{0}$ terms are balanced only if $c_{0}=b_{0}$ and in the next order we, neglecting the asymptotically small $O\left(\left(\frac{u_{*}}{U_{0}}\right)^{3}\right)$ contributions, obtain the differential equation:

$$
U_{0} \delta \frac{d u_{*}^{2}}{d x}=\alpha_{1} u_{*}^{3} \frac{d \delta}{d x}
$$


where $\alpha_{1}=2 \frac{b_{1}-c_{1}}{c_{0} \alpha_{0}}<0$ is a constant which must be obtained from a full local theory.

It is easy to see that the expression:

$$
\lambda=2\left(\frac{u_{*}}{U_{0}}\right)^{2}=\frac{\kappa}{\ln ^{2} \delta} ; \quad \lambda \propto \frac{d \delta}{d x}
$$

with $\kappa=8 / \alpha_{1}^{2}$ is a solution to (17). Indeed, integrating (17) and dividing the outcome by $U_{0}^{3}$, we obtain:

$$
\frac{\lambda}{2}=\frac{\left|\alpha_{1}\right| \sqrt{\kappa}}{4 \sqrt{2}} \int \frac{d \lambda}{d x} d x=\frac{\left|\alpha_{1}\right| \sqrt{\kappa}}{4 \sqrt{2}} \lambda
$$

This result shows that the anzatz (12)-(13) with $\lambda \propto \frac{1}{\ln ^{2} \delta}$ is a self-consistent solution to the Navier-Stokes -Prandtl equations of motion.

Setting for a time being all proportionality coefficients equal to unity, we introducing $\delta_{0}=\frac{\nu}{U_{0}}, R e_{\delta}=\frac{U_{0} \delta}{\nu}$ and $R e_{x}=\frac{U_{0} x}{\nu}$ solve the the differential equations (17) with the result: $R e_{\delta}\left[\left(\ln R e_{\delta}\right)^{2}-2 \ln \frac{R e_{\delta}}{e}\right]=R e_{x}$ leading to

$$
\delta(x) \propto \frac{x}{\ln ^{2} R e_{x}}
$$

in the limit $R e_{\delta} \rightarrow \infty$.

The derivation of asymptotic expressions (18)-(19) was based on the relation $\frac{d \delta}{d x} \propto\left(\frac{u_{*}}{U_{0}}\right)^{2} \propto \lambda$ which is obtained from (5) by neglecting the first contribution to the right side. With the scaling (6) this is equivalent to omitting $i_{1}$ in (7) as small. Now, based on the derived solution (18)(19) we justify this approximation. Indeed, comparing the integrands in the right side of (5) gives

$$
i_{1}=\delta\left(\frac{d}{d x} \frac{u_{*}}{U_{0}}\right) G=\frac{x}{\ln ^{2} R e_{x}} \frac{d \sqrt{\lambda}}{d x} G \approx \frac{G}{\ln ^{4} R e_{x}}
$$

and

$$
i_{2}=\frac{d \delta}{d x} \frac{u_{*}}{U_{0}} G \approx \frac{G}{\ln ^{3} R e_{x}}
$$

so that as $R e_{x} \rightarrow \infty$ the ratio $\frac{i_{1}}{i_{2}} \rightarrow 0$. Thus the term $i_{1}$ in (7) is asymptotically negligible.

Summary and discussion.

1. In this work the expression for skin friction in the zero-pressure-gradient boundary layer has ben derived directly from the Navier-Stokes equations. Similar results were recently presented in a resent paper ${ }^{3}$. In this work, to fit experimental data, the authors used a particular forth-order Pade approximant yielding logarithmic velocity profile in a leading order. This immediately gave $U_{+}(\delta) \approx \ln \delta_{+}$and the Coles-Fernholtz relation (1) for the skin friction. Since it has not been shown that the Pade approximant is an asymptotic solution to the Navier-Stokes equations, this theory is essentially semiempirical.

2. The theory presented here is different. No experimental information has been used to derive (18)-(19). Moreover, while we can say that these expressions are consistent with $U_{+} \propto \ln y_{+}$, the mathematical proof that this is so does not exist.

3. In arriving in the final result (18)-(19) we assumed that all scaling functions $\phi_{i}(\xi)$ are analytic. It is important to understand that in a logarithmic theory one can expect singularities when dealing with derivatives over spatial coordinates $x$ or $y$. It is not what has been done deriving the expansions (12)-(13). It is clear that the derivatives over the functions $\xi(x)=u_{*}(x) / U_{0}$ cannot be singular by definition: it is impossible to obtain a large response of, say, $U_{+}$upon infinitesimal variation of the friction velocity $u_{*}$. This simply contradicts all conservation laws.

4. The expression (18)-(19) for friction factor (skin friction) was found as a self-consistent solution to the Navier-Stokes-Prandtl equations. The full mathematical theory leading, in addition to the scaling relations derived here, to the amplitudes, remains an unsolved problem.

5 . These results are accurate up to the $O\left(u_{*} / U_{0}\right)$ corrections.

I am grateful to I. Staroselsky, S. Bailey, G. Falkovich, J. Schumacher, K.R. Sreenivasan, A. Smits, A. Polyakov, P. Monkewitz , N. Peters, C. Vassilicos, A. Yakhot and the students of the graduate turbulence course (BU, ME 709) for many informative and stimulating discussions.

${ }^{1}$ H. Schlichting, Boundary-Layer Theory, New York, NY, McGrow-Hill Book Company (1968).

${ }^{2}$ A.J. Smits, \& I. Marusic, High Reynolds number flows: a challenge for experiment and simulation, AIAA 99-3530 (1999).

${ }^{3}$ P.A. Monkewitz, K.A. Chauhan, \& H.M. Nagib Self-consistent high-Reynolds number asymptotics for zero-pressure-gradient boundary layers, Phys. Fluids 19, 115101 (2007).

${ }^{4}$ H.H. Fernholtz, \& P.J. Finley, The incompressible zero-pressure gradient turbulent boundary layer, Prog. Aerosp. Sci. 32, 245-211 (1966).

${ }^{5}$ J.O. Hinze, Turbulence, New York, NY, McGraw Hill Book Co. 1965.

${ }^{6} \mathrm{X}$. Wu \& P. Moin, A direct numerical simulation study on the mean velocity characteristics in turbulent pipe flow, J. Fluid Mech. 608, 81-112 (2008).

${ }^{7}$ V. Yakhot, S.C.C. Bailey \& A.J. Smits, A.J., Scaling of global properties of turbulence and skin friction in pipe and channel flows, J.Fluid Mech., 652, 65-73. (2010) 